\section{A novel technique for partial stent-in-stent placement of three metal biliary stents using a short double-balloon enteroscope}

The endoscopic partial stent-in-stent (PSIS) placement of self-expandable metal stents (SEMSs) is effective for the palliation of malignant hilar biliary strictures [1 -5]. Despite its efficacy, however, PSIS placement is technically challenging, especially when placing second or subsequent stents. We report a novel technique for PSIS placement of three SEMSs using a short double-balloon enteroscope (DBE), which was used in a patient with a malignant hilar biliary stricture and surgically altered anatomy.

A 74-year-old man who had undergone distal gastrectomy with Billroth II reconstruction was admitted with jaundice due to cholangiocarcinoma with a Bismuth type IV hilar biliary stricture
( Fig.1a). To aid biliary drainage, we placed a 7-Fr plastic stent in the left hepatic duct, another in the right posterior hepatic duct, and a third in the right anterior hepatic duct using a short DBE (EI-530B; Fujifilm, Tokyo; working channel, 2.8-mm diameter). Although this led to immediate resolution of the patient's jaundice, we diagnosed unresectable cholangiocarcinoma and therefore went on to perform PSIS placement of three SEMSs (Zilver 635; Cook Medical, Winston-Salem, North Carolina, USA) using the DBE before the patient commenced chemotherapy.

First, the stricture was dilated (Quantum, 6-mm diameter; Cook Medical) then, to identify the bifurcation of the common hepatic duct and the target bile duct, two 0.018-inch landmark guidewires (Roadrunner; Cook Medical) were inserted into the right posterior hepatic duct and the right anterior hepatic duct $[1,2]$. The first SEMS (10-mm diameter, $80-\mathrm{mm}$ long) was then placed into the left hepatic duct over a stiff 0.035-inch guidewire (THSF; Cook Medical) using a small-diameter (6-Fr), 200-cm-long delivery system, while keeping the two landmark guidewires in the right anterior and posterior hepatic ducts ( $\mathbf{F i g} \mathbf{1} \mathbf{b}$ ).

Next, a 0.035-inch hydrophilic guidewire (NaviPro; Boston Scientific, Natick, Massachusetts, USA) was easily inserted into the right posterior hepatic duct through the stricture and the interstices of the first SEMS following the landmark guidewire (๑ Fig. 1 c). A second SEMS (10-mm diameter, 60 -mm long) was then successfully placed in the right posterior hepatic duct using a stiff guidewire that had been exchanged for the hydrophilic guidewire ( Fig. 1d). The third SEMS (10-mm diameter, 60-mm long) was then placed
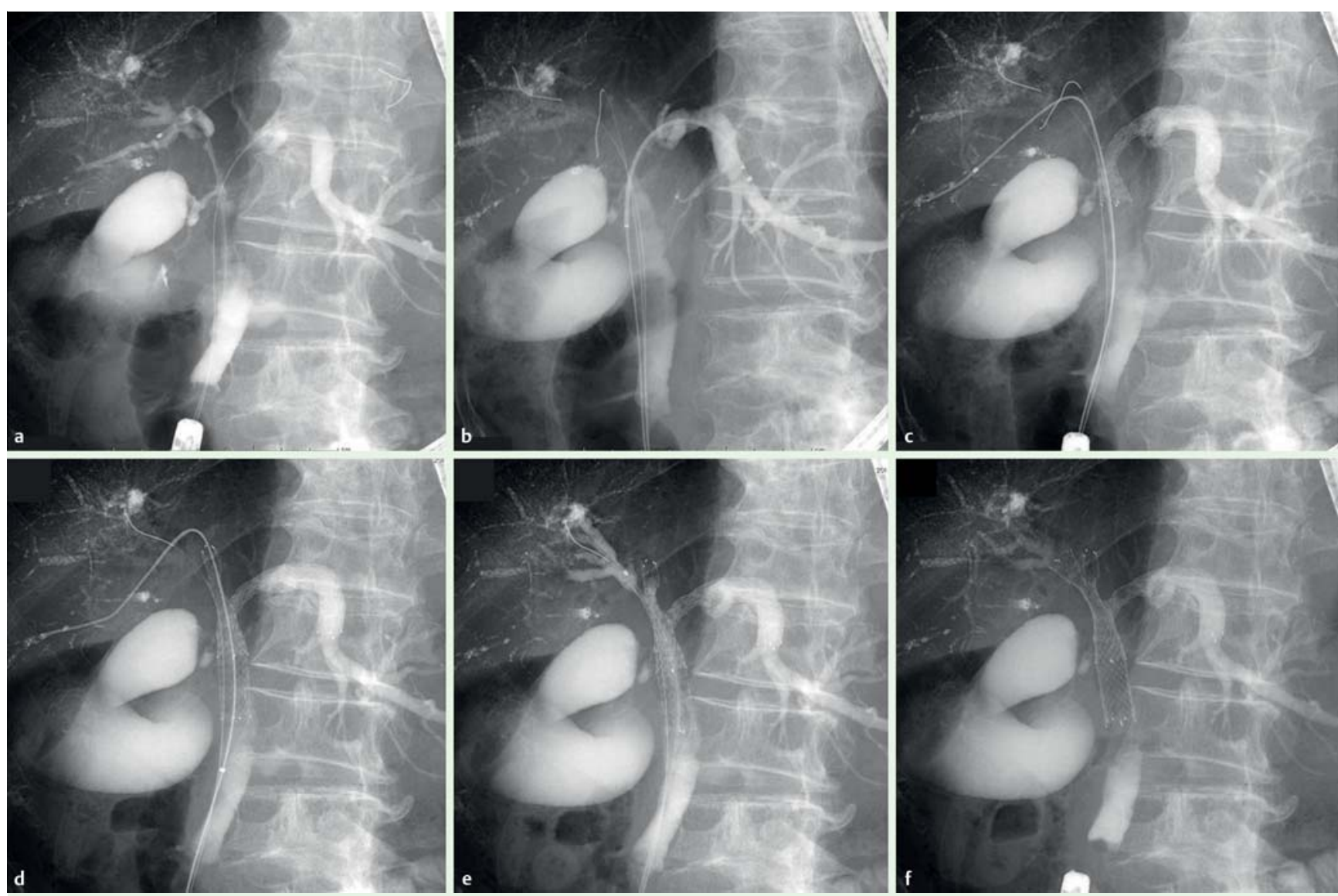

Fig. 1 Cholangiographic views using a short double-balloon enteroscope showing: a a Bismuth type IV malignant hilar biliary obstruction; b the two landmark guidewires in the right anterior and posterior hepatic ducts and the first stent, which was placed across the bifurcation into the left hepatic duct; $\mathbf{c}$ a 0.035 -inch hydrophilic guidewire passing into the right posterior hepatic duct through the stricture and the interstices of the first stent; $\mathbf{d}$ the second stent in the right posterior hepatic duct; e a guidewire passing through the interstices of the two previous stents and into the right anterior hepatic duct, over which the third stent was placed; $\mathbf{f}$ the three Zilver 635 stents forming a partial stent-in-stent arrangement. 
into the right anterior hepatic duct over a guidewire that had been passed in similar fashion through the interstices of the two previous stents ( Fig. 1 e,f).

Therefore, the combined use of the 6-Fr Zilver 635 SEMSs [5] and 0.018-inch landmark guidewires facilitated the PSIS placement of multiple SEMSs for malignant hilar biliary stricture using a short DBE with a small working channel.

Endoscopy_UCTN_Code_TTT_1AR_2AZ

Competing interests: None

Koichiro Tsutsumi, Hironari Kato, Hiroyuki Okada, Kazuhide Yamamoto

Department of Gastroenterology \& Hepatology, Okayama University Graduate School of Medicine, Dentistry, and Pharmaceutical Sciences, Okayama, Japan

\section{References}

1 Kawamoto H, Tsutsumi K, Fujii M et al. Endoscopic 3-branched partial stent-in-stent deployment of metallic stents in high-grade malignant hilar biliary stricture (with videos). Gastrointest Endosc 2007; 66: 1030 1037

2 Kato H, Tsutsumi K, Harada $R$ et al. Endoscopic bilateral deployment of multiple metallic stents for malignant hilar biliary strictures. Dig Endosc 2013; 25: 75-80

3 Tsutsumi K, Kato H, Tomoda T et al. Partial stent-in-stent placement of biliary metallic stents using a short double-balloon enteroscopy. World J Gastroenterol 2012; 18: 6674-6676

4 Chahal P, Baron TH. Expandable metal stents for endoscopic bilateral stent-within-stent placement for malignant hilar biliary obstruction. Gastrointest Endosc 2010; 71: $195-199$

5 Law R, Baron TH. Bilateral metal stents for hilar biliary obstruction using a 6Fr delivery system: outcomes following bilateral and side-by-side stent deployment. Dig Dis Sci 2013; 58: 2667-2672

\section{Bibliography}

DOI http://dx.doi.org/

10.1055/s-0034-1377408

Endoscopy 2014; 46: E417-E418

(c) Georg Thieme Verlag KG

Stuttgart · New York

ISSN 0013-726X

\section{Corresponding author}

\section{Koichiro Tsutsumi, MD}

Department of Gastroenterology Hepatology Okayama University Graduate School of Medicine, Dentistry, and Pharmaceutical Sciences

2-5-1 Shikata-cho, Kita-ku

Okayama-city

Okayama, 700-8558

Japan

Fax: +81-86-2255991

tsutsumi@cc.okayama-u.ac.jp 